\title{
WEAKENING THE TOPOLOGY OF A LIE GROUP
}

\author{
BY
}

T. CHRISTINE STEVENS

\begin{abstract}
With any topological group $(G, \mathscr{U})$ one can associate a locally arcwiseconnected group $\left(G, \mathscr{U}^{*}\right)$, where $\mathscr{U}^{*}$ is stronger than $\mathscr{U} .(G, \mathscr{U})$ is a weakened Lie $(W L)$ group if $\left(G, \mathscr{U}^{*}\right)$ is a Lie group. In this paper the author shows that the WL groups with which a given connected Lie group $(L, \mathscr{T})$ is associated are completely determined by a certain abelian subgroup $H$ of $L$ which is called decisive. If $L$ has closed adjoint image, then $H$ is the center $Z(L)$ of $L$; otherwise, $H$ is the product of a vector group $V$ and a group $J$ that contains $Z(L) . J / Z(L)$ is finite (trivial if $L$ is solvable). We also discuss the connection between these theorems and recent results of Goto.
\end{abstract}

1. Introduction. Gleason and Palais [1] have shown how to associate with any topological group $(G, \mathscr{U})$ a locally arcwise-connected group $\left(G, \mathscr{U}^{*}\right)$ and proved that the group thus associated with a finite-dimensional metric group must be Lie. In this paper we describe the groups for which the associated locally arcwise-connected group is a connected Lie group. As we shall see in $\$ 3$, this problem is equivalent to the following question: Given a connected Lie group $(L, \mathscr{T})$, in what ways can $\mathscr{T}$ be weakened and remain Hausdorff? Our principal result is that $L$ contains an abelian subgroup $H$ which is decisive in the sense that the ways in which $\mathscr{T}$ can be weakened and remain Hausdorff are completely determined by the ways in which the relative topology for $\boldsymbol{H}$ can be weakened while remaining Hausdorff and keeping finitely many characters of $H$ continuous. The nature of $H$ depends upon a crucial distinction between (CA) analytic groups (those with closed adjoint image) and non-(CA) analytic groups. Our proof in the latter case employs a homomorphism used by Goto [3] and relies upon structure theorems of Goto [4] and Zerling [18]. The connection between the present paper and certain results which were recently obtained by Goto [5] is discussed in §8. We also note that Hudson's examination of arcwise-connected, finite-dimensional groups [7] leads him to consider, from a different perspective, questions similar to those studied here.

2. Notation and conventions. A topology $\mathscr{U}$ for an abstract group $G$ will be assumed to make the function $f: G \times G \rightarrow G$ given by $f(x, y)=x y^{-1}$ continuous, but $\mathscr{U}$ need not be Hausdorff. For a subgroup $H$ of $G, \mathscr{U}_{H}$ will denote the relative topology

Presented to the Society by abstract on October 23, 1978, and at the Annual Meeting in Biloxi on January 25, 1979; received by the editors April 28, 1980 and, in revised form, July 13, 1981.

1980 Mathematics Subject Classification. Primary 22A05; Secondary 22E15, 22E25.

Key words and phrases. Lie group, locally arcwise-connected group, (CA) analytic group. 
induced by $\mathscr{U}$. Since several topologies for the same abstract group $G$ may be under simultaneous consideration, topological statements about $G$ will contain references to the particular topology involved. If $(G, \mathscr{U})$ is a topological group, then $T(G, \mathscr{U})$ will denote the collection of all Hausdorff group topologies for $G$ which are weaker than $\mathscr{U}$. Analytic groups, but not Lie groups, are always connected, and Lie groups do not necessarily satisfy the second axiom of countability.

$\mathbf{Z}, \mathbf{R}, T^{q}$, and $\mathrm{Gl}(V)$ denote, respectively, the integers, the real numbers, the $q$-dimensional toroid, and the linear automorphism group of a vector space $V . T^{q}$, $\mathrm{Gl}(V)$, and finite groups will be assumed to have their usual topologies, unless stated otherwise. The relative topology for a subgroup $A$ of $\mathrm{Gl}(V)$ will be called the fulllinear-group ( $f l g$ ) topology for $A$. The symbol $\square$ marks the end of a proof.

3. Preliminary definitions and main results. Let $(G, \mathscr{T})$ be a topological group. According to [1, pp. 634-635], the collection of all $\mathscr{T}$-arc-components of $\mathscr{T}$-open subsets of $G$ is the basis for a locally arcwise-connected group topology $\mathscr{T}^{*}$ for $G$. $\left(G, \mathscr{T}^{*}\right)$ is the locally arcwise-connected (l.a.c.) group associated with $(G, \mathscr{T})$. For easy reference, we list here some of the properties of l.a.c. groups that are proved in [1].

3.1. Theorem (Gleason and Palais $[1,3.2,4.3,7.3])$. Let $(G, \mathscr{T})$ be a Hausdorff topological group. Then

(i) $\mathscr{T}^{*}$ is stronger than $\mathscr{T}$, and $\left(\mathscr{T}^{*}\right)^{*}=\mathscr{T}^{*}$.

(ii) $(G, \mathscr{T})$ and $\left(G, \mathscr{T}^{*}\right)$ have the same arcs.

(iii) The $\mathscr{T}^{*}$-connected components of a $\mathscr{T}^{*}$-open subset X of $G$ are the $\mathscr{T}$-arc-components of $X$.

(iv) If $(G, \mathscr{T})$ is a second-countable Lie group and $\mathscr{U} \in T(G, \mathscr{T})$, then $\mathscr{U}^{*}=\mathscr{T}$.

(v) If $(G, \mathscr{T})$ is separable, metrizable, and of finite topological dimension, then $\left(G, \mathscr{T}^{*}\right)$ is a Lie group.

Hudson observes that the assumption of metrizability in (v) may be dropped if by "dimension" one means cohomological dimension [6, p. 68]. We will say that $(G, \mathscr{T})$ is a weakened Lie $(W L)$ group if $\left(G, \mathscr{T}^{*}\right)$ is a Lie group and $\mathscr{T}$ is Hausdorff. From (iv) we see that the WL groups with which a given analytic group $(L, \mathscr{T})$ is associated are all those of the form $(L, \mathscr{U})$, where $\mathscr{U} \in T(L, \mathscr{T})$.

We now introduce the notion of "decisiveness," which is central to our main result. Let $(A, \mathscr{A})$ be a topological group with an abstract subgroup $B$, and let $I(b)$ denote conjugation by an element $b$ of $B$. (Recall that group topologies are not assumed to be Hausdorff.) If $\mathscr{B}$ is a topology for $B$ that makes the function $f: A \times B \rightarrow A$, defined by $f(a, b)=b a b^{-1},(\mathscr{A} \times \mathscr{B}, \mathscr{A})$-continuous, then $\mathscr{A} \times \mathscr{B}$ is a group topology for the semidirect product $A \times{ }_{I} B$. We define a homomorphism $\alpha$ of $A \times{ }_{I} B$ onto $A$ by $\alpha(a, b)=a b$, and the unique topology for $A$ which makes $\alpha$ continuous and open is called the standard extension of $\mathscr{B}$ to $A$, denoted $\mathscr{E}(\mathscr{B})$. We observe that a basis for the neighborhoods of the identity in $\mathscr{E}(\mathscr{B})$ is the collection of all $P N$, where $P$ and $N$ are, respectively, $\mathscr{A}$ - and $\mathscr{B}$-neighborhoods of $e . \mathscr{E}(\mathscr{B})$ will be Hausdorff if $\mathscr{A}$ is Hausdorff, $B$ is $\mathscr{A}$-closed, and $\mathscr{B} \in T\left(B, \mathscr{A}_{B}\right)$.

When $\mathscr{B}$ is weaker than $\mathscr{A}_{B}, \mathscr{E}(\mathscr{B})$ may be thought of as "weakening the topology 
of $A$ by weakening that of $B$ "; we are interested in situations where this is the only way in which $\mathscr{A}$ can be weakened. If $B$ is $\mathscr{A}$-closed and if $\mathscr{U}=\mathscr{E}\left(\mathscr{U}_{B}\right)$ for every $\mathscr{U} \in$ $T(A, \mathscr{A})$, we will say that $B$ is decisive in $(A, \mathscr{A})$. We may now state our main result, the proof of which is contained in $\$ \S 4-6$.

3.2. MAIN THEOREM. Let $(L, \mathscr{T})$ be a connected Lie group with Lie algebra 1 .

(i) $L$ contains an abelian decisive subgroup $H$ of the form $\mathbf{R}^{p} \times T^{q} \times \mathbf{Z}^{r} \times D$, where $p, q$, and $r$ are nonnegative integers and $D$ is finite. The adjoint image of $H$ is contained in a toroid $Q$.

(ii) The WL groups with which $(L, \mathscr{T})$ is associated are precisely those of the form $(L, \mathscr{E}(\mathscr{B}))$, where $\mathscr{B} \in T\left(H, \mathscr{T}_{H}\right)$ and the restriction of the adjoint representation, Ad: $H \rightarrow Q$, is $\mathscr{B}$-continuous.

(iii) If $(L, \mathscr{T})$ is $(C A)$-that is, if $\operatorname{Ad}(L)$ is a flg-closed subgroup of $\mathrm{Gl}(l)$-then $H$ is the center of $L$.

(iv) If $(L, \mathscr{T})$ is not $(C A)$, then $H=J \times V$, where $V$ is a vector group, $J$ contains the center $Z(L)$ of $L$, and $J / Z(L)$ is finite (trivial if $L$ is solvable).

We conclude this section with an important lemma about standard extensions.

3.3. LeMMA. Let $(A, \mathscr{A})$ be a topological group with an abstract subgroup $B$. Let $\mathscr{C}$ be the collection of all topologies $\mathscr{A}^{\prime}$ for $A$ that are weaker than $\mathscr{A}$ and such that the function $h: A \times A \rightarrow A$ given by $h(a, g)=g a g^{-1}$ is $\left(\mathscr{A} \times \mathscr{A}^{\prime}, \mathscr{A}\right)$-continuous. If $\mathscr{U} \in \mathscr{C}$ and $\mathscr{U}=\mathscr{E}\left(\mathscr{U}_{B}\right)$, then $\mathscr{A}^{\prime}=\mathscr{E}\left(\mathscr{A}_{B}^{\prime}\right)$ for every $\mathscr{A}^{\prime}$ in $\mathscr{C}$ which is stronger than $\mathscr{U}$.

Proof. By hypothesis, the homomorphism $\alpha: A \times{ }_{I} B \rightarrow A$ given by $\alpha(a, b)=a b$ is $\left(\mathscr{A} \times \mathscr{U}_{B}, \mathscr{U}\right)$-open and continuous. Let $\mathscr{A}^{\prime} \in \mathscr{C}$ be stronger than $\mathscr{U}$, and let $\left\{g_{i}: i \in I\right\}$ be a net in $A$ which $\mathscr{A}^{\prime}$-converges to $e$. Then $\left\{g_{i}\right\}$ also $\mathscr{U}$-converges to $e$ and thus has a subnet $\left\{g_{i(j)}: j \in J\right\}$ which lifts to a net $\left\{\left(a_{j}, b_{j}\right): j \in J\right\}$ in $A \times{ }_{I} B$ that is $\mathscr{A} \times \mathscr{U}_{B^{-}}$ convergent to $e$. Since $b_{j}=a_{j}^{-1} g_{i(j)}$ and $\mathscr{A}^{\prime}$ is weaker than $\mathscr{A}$, it follows that $b_{j} \rightarrow e$ in $\mathscr{A}^{\prime}$, and thus $\alpha$ is $\left(\mathscr{A} \times \mathscr{A}_{B}^{\prime}, \mathscr{A}^{\prime}\right)$-open.

4. Decisive subgroups. The purpose of this section is to prove the first three parts of Main Theorem 3.2; the proof of part (iv) is reserved for $\S \S 5$ and 6 . We begin by proving that every WL group has a continuous adjoint representation.

Let $(G, \mathscr{U})$ be a WL group, and let $L$ be the $\mathscr{U}$-arc-component of the identity. By $3.1,\left(L,\left(\mathscr{U}^{*}\right)_{L}\right)$ is an analytic group (whose Lie algebra we denote by $\left.l\right)$ and $\left(\mathscr{U}^{*}\right)_{L}=$ $\left(\mathscr{U}_{L}\right)^{*}$. $G$ acts by inner automorphism on $L$, and we denote by $I$ and Ad the corresponding homomorphisms of $G$ into $\operatorname{Aut}(L)$ and $\operatorname{Gl}(l)$. If $\operatorname{Aut}(L)$ has the generalized compact-open topology, then $I$ and Ad are $\mathscr{U}^{*}$-continuous. We now show that they are also $\mathscr{U}$-continuous.

4.1. Lemma. Let $\lambda:[0,1] \rightarrow L$ be a $\mathscr{U}_{L}^{*}$-arc with initial point $e$ and let $\left\{x_{j}: j \in J\right\}$ be $a$ net in $G$ which $\mathscr{U}$-converges to $x \in G$. Then $\left\{x_{j} \lambda(t) x_{j}^{-1}: j \in J\right\} \mathscr{U}_{L}^{*}$-converges to $x \lambda(t) x^{-1}$, uniformly in $t$.

Proof. Let $N$ be the $\mathscr{U}_{L}$-arc-component of $e$ in a $\mathscr{U}_{L}$-open neighborhood $U$ of $e$, and let $U_{1}$ be a $\mathscr{U}_{L}$-open neighborhood of $e$ such that $U_{1}^{2} \subseteq U$. For each $j \in J$, define 
a $\mathscr{U}_{L}$-arc $\beta_{j}:[0,1] \rightarrow L$ by

$$
\beta_{j}(t)=x \lambda(-t) x^{-1} x_{j} \lambda(t) x_{j}^{-1} .
$$

For each $s$ in $[0,1]$, there exist an open neighborhood $I_{s}$ of $s$ and an element $j_{s}$ of $J$ such that

$$
x \lambda(-s) x^{-1} x_{j} \lambda(t) x_{j}^{-1} \in U_{1}
$$

and

$$
x \lambda(-t) \lambda(s) x^{-1} \in U_{1}
$$

whenever $t \in I_{s}$ and $j \geq j_{s}$. From (1) and (2) we see that $\beta_{j}(t)$ is in $U$ if $t \in I_{s}, j \geq j_{s}$. By compactness of the unit interval, there is a $j^{*} \in J$ such that $\beta_{j}(t) \in U$ for all $t \in[0,1]$ and for all $j \geq j^{*}$. For such $j, \beta_{j}$ will then be an arc in $U$ that contains the identity, and therefore $\beta_{j}$ is in fact an $\operatorname{arc}$ in $N$. Thus $\beta_{j}(t) \in N$ for all $t \in[0,1]$ and for all $j \geq j^{*}$, and the lemma is proved.

\subsection{Proposition. Ad: $G \rightarrow \mathrm{Gl}(l)$ and $I: G \rightarrow \operatorname{Aut}(L)$ are $\mathscr{U}$-continuous.}

Proof. Let $\left\{x_{j}: j \in J\right\}$ be a net in $G$ which $\mathscr{U}$-converges to $e$, and let $W$ be an open neighborhood of 0 in $l$ such that the exponential mapping exp: $l \rightarrow L$ is a diffeomorphism on $2 W$. To prove that $\operatorname{Ad}$ is $\mathscr{U}$-continuous, it is sufficient to show that $\operatorname{Ad}\left(x_{j}\right)(w)$ $\rightarrow w$ for any $w \in W$. From 4.1 we know that there is an element $j^{*}$ of $J$ such that, if $j \geq j^{*}$, then

$$
\exp \left(t \operatorname{Ad}\left(x_{j}\right)(w)\right)=x_{j}(\exp t w) x_{j}^{-1} \in \exp W
$$

for all $t \in[0,1]$, and it follows that $\operatorname{Ad}\left(x_{j}\right)(w) \in W$ if $j \geq j^{*}$. Lemma 4.1 also implies that, in $U_{L}^{*}$,

$$
\exp \left(\operatorname{Ad}\left(x_{j}\right)(w)\right)=x_{j}(\exp w) x_{j}^{-1} \rightarrow \exp w .
$$

Since exp is a diffeomorphism on $W$, we conclude that $\operatorname{Ad}\left(x_{j}\right)(w) \rightarrow w$ in $l$, as desired.

To prove that $I$ is $\mathscr{U}$-continuous, we simply observe that the differential operator $d$ is a topological group isomorphism of $\operatorname{Aut}(L)$ onto a subgroup of $\mathrm{Gl}(l)$. This completes the proof of 4.2 .

We now deduce an important criterion by which decisive subgroups of analytic groups may be identified.

4.3. LEMMA. Let $(L, \mathscr{T})$ be an analytic group, $B$ a $\mathscr{T}$-closed subgroup of $L$, and $\mathscr{U}$ the weakest (not necessarily Hausdorff) topology for $L$ that makes Ad $\mathscr{U}$-continuous. If $\mathscr{U}=\mathscr{E}\left(\mathscr{U}_{B}\right)$, then $B$ is decisive in $L$.

Proof. According to 3.1 and 4.2, every topology in $T(L, \mathscr{T})$ is stronger than $\mathscr{U}$. We may then apply 3.3 , with $A=L$ and $\mathscr{A}=\mathscr{T}$.

If $(L, \mathscr{T})$ is a (CA)analytic group, then $\operatorname{Ad}(L)$ is closed in $\mathrm{Gl}(l)$. If $(L, \mathscr{T})$ is not(CA), then $\operatorname{Gl}(l)$ contains a toral subgroup $Q$ such that the flg-closure $C$ of $\operatorname{Ad}(L)$ equals $\operatorname{Ad}(L) \cdot Q$. (See, for example, Goto [2, Theorem 1].) Thus in either case, $C=\operatorname{Ad}(L) \cdot Q$, where $Q$ is a (possibly trivial) toral subgroup of $\mathrm{Gl}(l)$. 
4.4. Proposition. Let $(L, \mathscr{T})$ be an analytic group with Lie algebra l, let $C$ be the flg-closure of $\operatorname{Ad}(L)$, and let $Q$ be a (possibly trivial) toral subgroup of $\mathrm{Gl}(l)$ such that $C=\operatorname{Ad}(L) \cdot Q$. Then $\operatorname{Ad}^{-1}(Q)$ is an abelian decisive subgroup of $L$ and has the form $\mathbf{R}^{p} \times T^{q} \times \mathrm{Z}^{r} \times D$, where $p, q$, and $r$ are nonnegative integers and $D$ is finite.

Proof. Let $H=\operatorname{Ad}^{-1}(Q)$. Because $d: \operatorname{Aut}(L) \rightarrow d(\operatorname{Aut}(L))$ is a topological isomorphism whose image is fig-closed and includes $\operatorname{Ad}(L)$, there is a toral subgroup $K$ of Aut $(L)$ such that $Q=d K$, and thus $H=I^{-1}(K)$. According to Goto [3, Lemma 4], each automorphism in $K$ leaves $H$ pointwise fixed. In particular, since $I(H) \subseteq K$, $H$ is abelian and therefore has the form $\mathbf{R}^{p} \times T^{q} \times E$, where $p$ and $q$ are nonnegative integers and $E$ is discrete. We now invoke Theorem $1^{\prime}$ and the subsequent remark in Mostow [8] to show that $E$ is finitely generated and thus equals $Z^{r} \times D$, where $r$ is a nonnegative integer and $D$ is finite.

It remains to show that $H$ is decisive in $L$. If $\mathscr{U}$ is the weakest topology for $L$ which makes Ad ( $\mathscr{U}$, flg)-continuous, then it suffices, by 4.3 , to show that $\mathscr{U}=\mathscr{E}\left(\mathscr{U}_{H}\right)$. To do so, we form the semidirect product $L$ (s) $K$ and define a homomorphism $\psi: L$ (s) $\rightarrow C$ by $\phi(x, k)=\operatorname{Ad}(x) \cdot d k$ for $x \in L, k \in K$. If $\mathscr{W}$ is the topology which $K$ inherits from the generalized compact-open topology, then $\psi$ is surjective, $(\mathscr{T} \times \mathscr{W}$, flg)-continuous, and thus $(\mathscr{T} \times \mathscr{W}$, flg)-open onto its image $C$. It follows that a basis for the neighborhoods of $e$ in the flg-topology for $C$ is the collection of all $\operatorname{Ad}(P) \cdot d N$, where $P$ is a $\mathscr{T}$-neighborhood of $e$ in $L$ and $N$ is a $\mathscr{W}$-neighborhood of $e$ in $K$, and therefore a basis for the $\mathscr{U}$-neighborhoods of $e$ is the collection of all $\operatorname{Ad}^{-1}(\operatorname{Ad}(P) \cdot d N)$. Now for each such $P$ and $N$, the fact that Ad is a homomorphism implies that

$$
\operatorname{Ad}^{-1}(\operatorname{Ad}(P) \cdot d N)=P \cdot \operatorname{Ad}^{-1}(d N) .
$$

Since $\operatorname{Ad}^{-1}(d N)$ is simply a $\mathscr{U}_{H}$-neighborhood of $e$, we have shown that $\mathscr{U}=\mathscr{E}\left(\mathscr{U}_{H}\right)$.

Proposition 4.4 proves part (i) of the Main Theorem, and we may now proceed to prove parts (ii) and (iii). Let $H$ be any abelian decisive subgroup of $(L, \mathscr{T})$. We will call a topology $\mathscr{B}$ for $H$ allowable if $\mathscr{B} \in T\left(H, \mathscr{T}_{H}\right)$ and the restriction Ad: $H \rightarrow \mathrm{Gl}(l)$ is $\mathscr{B}$-continuous. If $(L, \mathscr{U})$ is a Hausdorff topological group and $\mathscr{U}^{*}=\mathscr{T}$, then $\mathscr{U}=$ $\mathscr{E}\left(\mathscr{U}_{H}\right)$, and by $4.2 \mathscr{U}_{H}$ is an allowable topology for $H$. On the other hand, if $\mathscr{B}$ is such a topology then $\mathscr{E}(\mathscr{B})$ is a group topology for $L$ which is in $T(L, \mathscr{T})$ and by 3.1 (iv) $(L, \mathscr{T})$ is the 1.a.c. group associated with $(L, \mathscr{E}(\mathscr{B}))$. To prove part (iii) of 3.2, we simply note that when $(L, \mathscr{T})$ is (CA) we may choose the trivial toroid for $Q$, so that the decisive subgroup $\operatorname{Ad}^{-1}(Q)$ will be the center of $L$.

5. Subgroups of $\operatorname{Gl}(n, \mathbf{R})$. Before proving part (iv) of the Main Theorem, which is the purpose of $\S 6$, we must examine in detail the structure of the flg-closure of $\operatorname{Ad}(L)$ when $(L, \mathscr{T})$ is not $(C A)$. The basis for our discussion is a result in Goto [4], which we now describe. Let $(G, \mathscr{U})$ be an analytic subgroup of $\mathrm{Gl}(n, \mathbf{R})$ which is not flg-closed, with $C$ denoting the flg-closure of $G$. Let $N$ be any subgroup of $G$ which is maximal among those that are $\mathscr{U}$-connected, fig-closed, and contain the commutator subgroup $D$ of $G$. (Such groups exist because, by Lemma 7 in [2], $D$ is flg-closed.) If $T_{1}$ is the radical of a maximal flg-compact subgroup of $C$, let $T_{2}$ be the flg-connected component of the identity in $N \cap T_{1}$ and $T$ a toroid such that $T_{1}=T_{2} \cdot T, T_{2} \cap T=\{e\}$. 
In our notation, Goto's theorem may be stated as follows:

5.1. Theorem (Goto [4, P. 197]). $N \cap T$ is finite, $C=N \cdot T$, and $G$ contains $a$ U-closed vector subgroup $W$, the flg-closure of which is $T$, such that $G=N \cdot W, N \cap W$ $=\{e\} . C$ is $\left(f l g, f g \times \mathscr{U}_{N}\right)$-djffeomorphic with $T \times N$.

Although the groups $N$ and $T$ are not in general unique, we will prove the following.

5.2. Proposition. If $N$ and $T$ are chosen in the manner described, then

(i) the dimensions of $N, W$, and $T$ are uniquely determined;

(ii) the finite groups $N \cap T$ are all isomorphic;

(iii) $N \cap T$ is trivial if $G$ is solvable.

The proposition depends upon a lemma about abelian analytic groups, the straightforward proof of which we omit.

5.3. LEMMA. Let $(A, \mathscr{A})$ be an abelian analytic group with a dense analytic subgroup $(B, \mathscr{B})$. Let $C$ be a maximal $\mathscr{B}$-connected and $\mathscr{A}$-closed subgroup of $B$. Then the dimension of $C$ equals the sum of the dimensions of the vector part of $(A, \mathscr{A})$ and the compact part of $(B, \mathscr{B})$, and the intersection of $C$ with the compact part of $(A, \mathscr{A})$ is independent of the choice of $C$.

Proof of 5.2. Let $\mathscr{U}^{\prime}$ and $\mathscr{F}^{\prime}$ denote the quotient topologies for $G / D$ and $C / D$ obtained from $\mathscr{U}$ and the flg-topology. To prove (i) it will suffice to show that $N / D$ is maximal among the subgroups of $G / D$ which are $\mathscr{U}^{\prime}$-connected and $\mathscr{F}^{\prime}$-closed, for then 5.3 will assure that the dimension of $N / D$, and thus of $N$, is uniquely determined $\left(N / D\right.$ must be $\mathscr{F}^{\prime}$-closed because it is the inverse image of the identity under the projection $C / D \rightarrow C / N$, which is continuous if each group is given the quotient topology from the flg-topology.) That $N / D$ is, in fact, maximal follows from the maximality of $N$ and the $\mathscr{U}$-connectedness of $D$.

To prove (ii), we first show that the intersection $N \cap T_{1}$ is, for given $T_{1}$, independent of the choice of $N$. For if $N_{1}$ and $N_{2}$ are two choices for $N$, then $N_{1} / D$ and $N_{2} / D$ are, as we have seen, maximal among the $\mathscr{U}^{\prime}$-connected subgroups of $G / D$ which are $\mathscr{F}^{\prime}$-closed, and so 5.3 implies that the intersections of $N_{1} / D$ and $N_{2} / D$ with the image of $T_{1}$ in $C / D$ are equal. Therefore $N_{1} \cap D T_{1}=N_{2} \cap D T_{1}$, whence $N_{1} \cap T_{1}=N_{2} \cap$ $T_{1}$.

Since $T_{1}$, as the radical of a maximal flg-compact subgroup of $C$, is determined up to conjugation by an element of $C$, and since $N$ is normal in $C$, the groups $N \cap T_{1}$ are thus all fig-isomorphic, regardless of the choices of $N$ and $T_{1}$. We complete the proof of (ii) by showing that, for fixed $N$ and $T_{1}$, the isomorphism class of the finite group $N \cap T$ is independent of the choice of $T$. This follows from the fact that, for any choice of $T, N \cap T_{1}=T_{2}(N \cap T)$ and $T_{2} \cap(N \cap T)=\{e\}$.

Finally, we prove (iii). It clearly suffices to show that $N \cap T_{1}$ is flg-connected when $G$ is solvable. Since $G$ and $C$ have the same commutator subgroup $D, C$ also is solvable. Applying Proposition 2.4 of Van Est [17], we find that $G=B \cdot A$, where $B$ is a flg-closed and simply-connected group containing $D$, the flg-closure $F$ of $A$ is a toroid, $C=B \cdot F$, and $B \cap F=\{e\}$. We may choose $N$ and a maximal flg-compact subgoup 
$K$ of $C$ in such a way that $B \subseteq N, F \subseteq K$. That the latter inclusion is in fact an equality follows from the easily verified equation $K=(B \cap K) \cdot F$ and the fact that $B$, as a simply-connected and solvable Lie group in the fig-topology, contains no nontrivial compact subgroups. We may therefore let $T_{1}$ equal $F$, and the proof is completed by observing that $N \cap F$ must be flg-connected, since $N=B(N \cap F), N$ and $B$ are fig-connected, and $B \cap(N \cap F)=B \cap F$ is trivial.

Applying 5.1 and 5.2 to the adjoint image of a non-(CA) analytic group, we obtain the following.

5.4. Corollary. Let $(L, \mathscr{T})$ be a non- $(C A)$ analytic group, $\mathscr{U}$ the Lie topology for $\operatorname{Ad}(L)$, and $C$ the flg-closure of $\operatorname{Ad}(L)$. If $N$ is a maximal $\mathscr{U}$-connected and flg-closed subgroup of $\operatorname{Ad}(L)$ containing the commutator subgroup of $\operatorname{Ad}(L), T_{1}$ is the radical of a maximal flg-compact subgroup of $C$, and $T$ is a toroid in $T_{1}$ complementary to the flg-connected component of the identity in $N \cap T_{1}$, then $\operatorname{Ad}(L)$ contains a $\mathscr{U}$-closed vector subgroup $W$, whose flg-closure is $T$, such that $\operatorname{Ad}(L)=N \cdot W, C=N \cdot T$, and $N \cap W$ is trivial. Moreover, $N \cap T$ is a finite group whose isomorphism class does not depend on the particular choices of $N$ and $T . N \cap T$ is trivial if $\operatorname{Ad}(L)$ is solvable, and the dimensions of $N, W$, and $T$ are independent of the choices of $N$ and $T$.

6. The non-(CA) case. The proof of part (iv) of the Main Theorem, which is contained in this section, relies not only upon 5.4 but also upon Zerling's structure theorem for non-(CA) analytic groups [18], which says that every such group is the semidirect product of a (CA) analytic group and a vector group. More precisely, the relevant portion of Zerling's results may be stated as follows.

6.1. Theorem (Zerling [18, Theorem 2.1]). With notation as in 5.4, let $P$ be the $\mathscr{T}$-connected component of the identity in $\operatorname{Ad}^{-1}(N)$. Then $\left(P, \mathscr{T}_{P}\right)$ is $(C A)$ and contains the center of $L$, and $\operatorname{Aut}(P)$ contains a vector subgroup $V$ with compact closure such that, if $\mathscr{V}$ is thevector topology for $V$, then $P(S)$ is $\left(\mathscr{T}_{P} \times \mathscr{V}, \mathscr{T}\right)$-isomorphic with $L$. If we identify $L$ with $P$ (S) $V$, then $\operatorname{Ad}(V)=W$, and $V$ and $W$ have the same dimension.

Adopting the notation of 5.4 and 6.1, we now combine these results with 4.4 to prove part (iv) of the Main Theorem. Since $C=\operatorname{Ad}(L) \cdot T$ by 5.4 , it follows from 4.4 that $H=\operatorname{Ad}^{-1}(T)$ is an abelian decisive subgroup of $L$. If we let $J$ equal $P \cap$ $\operatorname{Ad}^{-1}(N \cap T)$, a trivial computation verifies that $H=J$ (S) $V$. Since $H$ is abelian, $V$ must act trivially on $J$, whence $H=J \times V$. To complete the proof, we note that $\operatorname{Ad}(P)$ must equal all of $N$, since $N \cap \operatorname{Ad}(V)=N \cap W$ is trivial. Therefore $\operatorname{Ad}(J)=$ $N \cap T$ and $J / Z(L)$ is isomorphic to the finite group $N \cap T$. If $L$ is solvable, then so is $\operatorname{Ad}(L)$, and 5.4 assures that $J / Z(L)$ is trivial. This completes the proof of the Main Theorem. We may also note that, according to 5.4 and 6.1, the dimension of $V$ and the isomorphism class of $J / Z(L)$ are independent of the particular choices of $N$ and $T$.

7. Examples. We now give an example of a non-(CA) analytic group whose center, in the notation of the Main Theorem, has index two in the group $J$. Define an action of $S U(2) \times T^{2}$ on $\mathrm{C}^{3}$ by letting $\left(A, e^{i \theta}, e^{i \psi}\right)$, where $A \in S U(2)$, correspond to the $3 \times 3$ complex matrix in Figure 1. The semidirect product $G=C^{3}(S)\left(S U(2) \times T^{2}\right)$ 
is then a Lie group in its usual topology. If $\mu$ is some fixed irrational number, then $L=\mathbf{C}^{3}$ (S) $(S U(2) \times \mathbf{R})$ becomes a dense Lie subgroup of $G$ by means of the injection $\alpha:(z, A, t) \mapsto\left(z, A, e^{i t}, e^{i \mu t}\right), z \in \mathbf{C}^{3}, A \in S U(2), t \in \mathbf{R}$. $L$ has trivial center and thus cannot, by Theorem 2.2.1 of Van Est [16], be (CA).

By considering the action of $G$ on $L$ by inner automorphism, one verifies that $G / Z(G)$ is, in the quotient topology, isomorphic with the flg-closure of $\operatorname{Ad}(L)$, so that we may regard the adjoint representation of $L$ as the composition of $\alpha$ with the projection $\pi: G \rightarrow G / Z(G)$. Letting $N=\pi\left(C^{3} \times S U(2) \times 1 \times 1\right)$ and $T_{1}=$ $\pi\left(0 \times I \times T^{2}\right)$, we find that $N \cap T_{1}$ is a two-element group. Therefore $T=T_{1}$ and $\operatorname{Ad}^{-1}(T)=0 \times J \times \mathbf{R}$, where $J$ is the group generated by $-I$.

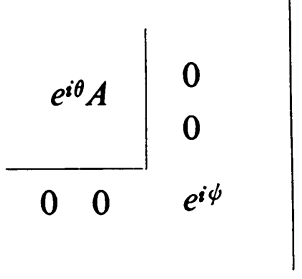

Figure 1

We also observe that the reduction to the abelian case which the Main Theorem effects does not prevent WL groups from having rather peculiar topologies. Although the author will undertake a systematic study of "unusual" topologies for abelian groups in a subsequent paper [15], we may note here that any sequence in $\mathbf{R}^{n}$ or $\mathbf{Z}^{n}$ which "goes to infinity sufficiently fast" will, in an appropriately weakened topology, converge to 0 . For example,

$$
d(n, m)=\inf \left\{\Sigma\left|c_{i}\right| / i \mid n-m=\Sigma c_{i}(i !+1), c_{i} \in \mathbf{Z}\right\}
$$

defines a metric on $\mathbf{Z}$ in which $i !+1 \rightarrow 0$. Other examples of unusual topologies for $\mathbf{Z}$ and $\mathbf{R}^{n}$ can be found in [9-14].

8. Related results. After writing this paper, the author learned of related but independent results obtained by Goto [5], and in this section we will sketch the connection between his work and ours. Let $(L, \mathscr{T})$ and $(G, \mathscr{U})$ be, respectively, an analytic and a topological group, and let $f: L \rightarrow G$ be a continuous, injective homomorphism. Although the course of Goto's analysis parallels our own in certain respects, his primary interest is a description of the set $\overline{f(L)}$, while our Main Theorem can be viewed as a characterization of the topology of $f(L)$. Changing Goto's notation to distinguish the $g m$-torus in [5] from our own decisive subgroup $H$, we can summarize his principal

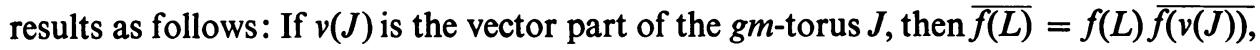
and $f$ is an imbedding (i.e., a homeomorphism of $L$ onto $f(L)$ ) if and only if $f \mid v(J)$ is. We note that the equation $\overline{f(L)}=f(L) \overline{f(v(J))}$ is a statement about sets, not topologies, and that it is trivially valid when $f(L)=G$.

Now our decisive subgroup $H$, besides revealing whether $f$ is an imbedding, also 
explicitly determines the topology of $f(L)$ when $f$ is not an imbedding; identifying $L$ with $f(L)$, we know that $\mathscr{U}_{L}=\mathscr{E}\left(\mathscr{U}_{H}\right)$. The following example illustrates the relationship among $H, J$, and $v(J)$. If $L=\mathbf{R} \times T^{1} \times S U(2)$, then $H=\mathbf{R} \times T^{1} \times\{ \pm I\}, J=$ $\mathbf{R} \times T^{1} \times O(2)$, and $v(J)=\mathbf{R} \times 1 \times I$. As the author will show in [15], there is a topology $\mathscr{U}$ for $L$, weaker than the usual topology, in which $\left\{\left(n !,(-1)^{n}, I\right)\right\}$ converges to the identity. Clearly $\mathscr{U}$ cannot equal $\mathscr{E}\left(\mathscr{U}_{v(J)}\right)$, and thus $v(J)$ is "too small" to determine the topology of $L$; indeed, a theorem in [15] shows that in this case $H$ is the smallest subgroup that will suffice. On the other hand, $J$ is "too big", because it properly contains $H$.

Finally, we note that Theorem 1 in [5] provides a somewhat more elegant proof of our 4.2 and that Theorem 2 in [5] is a sharper form of the theorem in [4] which we cited in $\$ 5$. The latter makes possible the following improvement in our Main Theorem: When $L$ is not (CA), we can alter the choice of $Q$ to assure that $H=Z(L) \times V$, even if $L$ is not solvable. The details are contained in [15].

\section{REFERENCES}

1. A. Gleason and R. Palais, On a class of transformation groups, Amer. J. Math. 79 (1957), 631-648.

2. M. Goto, Faithful representations of Lie groups. I, Math. Japon. 1 (1948), 107-119.

3. - Absolutely closed Lie groups, Math. Ann. 204 (1973), 337-341.

4. —_, Analytic subgroups of $\mathrm{Gl}(n, R)$, Tôhoku Math. J. (2) 25 (1973), 197-199.

5. — Immersions of Lie groups, J. Math. Soc. Japan 82 (1980), 727-749.

6. S. Hudson, On connectivity properties of finite-dimensional groups, Proc. Amer. Math. Soc. 23 (1969), 68-72.

7. - On the topology and geometry of arcwise connected finite-dimensional groups, Pacific J. Math. 82 (1979), 429-450.

8. G. Mostow, The fundamental group of a homogeneous space, Ann. of Math. 66 (1957), 249-255.

9. J. Nienhuys, Not locally compact monothetic groups. I, II, Nederl. Akad. Wetensch. Proc. Ser. A 73 = Indag. Math. 32 (1970), 295-326.

10. - A solenoidal and monothetic minimally almost periodic group, Fund. Math. 73 (1971/72), 167-169.

11. - Construction of group topologies on abelian groups, Fund. Math. 75 (1972), 101-116.

12. - Some examples of monothetic groups, Fund. Math. 88 (1975), 163-171.

13. H. Omori, Some examples of topological groups, J. Math. Soc. Japan 18 (1966), 147-153.

14. S. Rolewicz, Some remarks on monothetic groups, Colloq. Math. 13 (1964), 27-28.

15. T. C. Stevens, Decisive subgroups of analytic groups, Trans. Amer. Math. Soc. (to appear)

16. W. Van Est, Dense imbeddings of Lie groups, Nederl. Akad. Wetensch. Proc. Ser. A $54=$ Indag. Math 13 (1951), 321-328.

17. - Dense imbeddings of Lie groups. II, Nederl. Akad. Wetensch. Proc. Ser. A 55 = Indag. Math 14 (1952), 255-274.

18. D. Zerling, Some theorems on (CA) analytic groups, Trans. Amer. Math. Soc. 205 (1975), 181-192.

Department of Mathematics, Mount Holyoke College, South Hadley, Massachusetts 01075

Current address: Department of Mathematics and Physics, Arkansas State University, State University, Arkansas 72467 